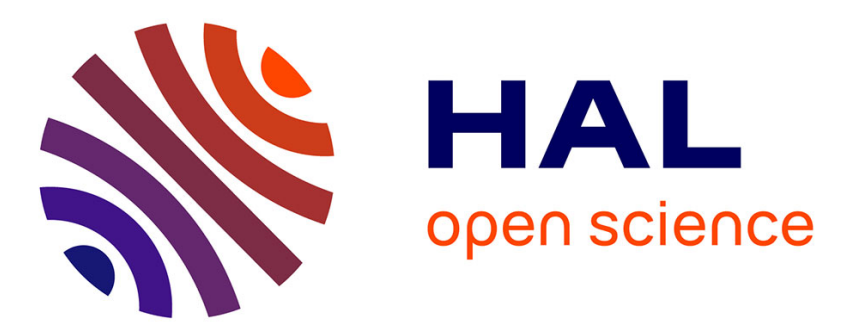

\title{
Numerical investigation of the combined effects of curvature and normal stress on sheet metal formability
}

Mohamed Ben Bettaieb, Farid Abed-Meraim, Xavier Lemoine

\section{To cite this version:}

Mohamed Ben Bettaieb, Farid Abed-Meraim, Xavier Lemoine. Numerical investigation of the combined effects of curvature and normal stress on sheet metal formability. International Journal of Material Forming, 2019, 12 (2), pp.211-221. 10.1007/s12289-017-1382-3 . hal-01797168

\section{HAL Id: hal-01797168 \\ https://hal.science/hal-01797168}

Submitted on 22 May 2018

HAL is a multi-disciplinary open access archive for the deposit and dissemination of scientific research documents, whether they are published or not. The documents may come from teaching and research institutions in France or abroad, or from public or private research centers.
L'archive ouverte pluridisciplinaire HAL, est destinée au dépôt et à la diffusion de documents scientifiques de niveau recherche, publiés ou non, émanant des établissements d'enseignement et de recherche français ou étrangers, des laboratoires publics ou privés. 


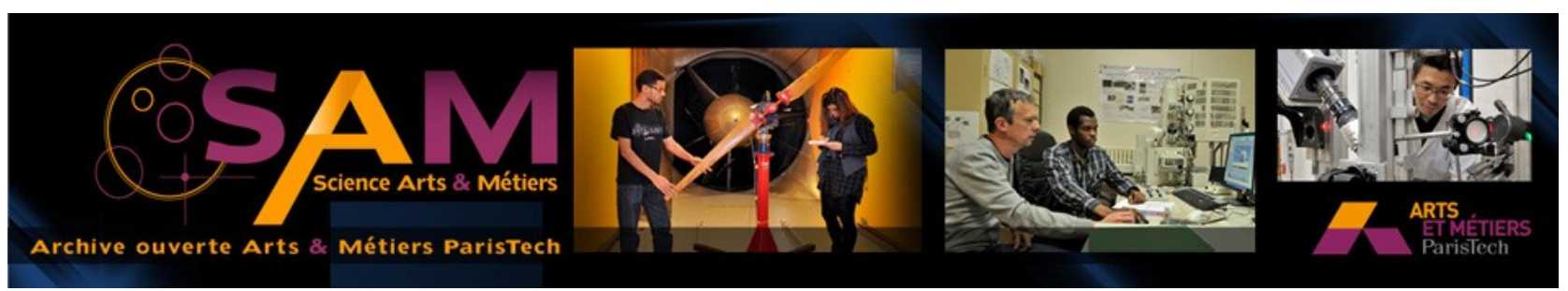

\section{Science Arts \& Métiers (SAM)}

is an open access repository that collects the work of Arts et Métiers ParisTech researchers and makes it freely available over the web where possible.

This is an author-deposited version published in: http://sam.ensam.eu Handle ID: .http://hdl.handle.net/null

\section{To cite this version :}

Mohamed BEN BETTAIEB, Farid ABED-MERAIM, Xavier LEMOINE - Numerical investigation of the combined effects of curvature and normal stress on sheet metal formability - International Journal of Material Forming p.1-11 - 2017 


\title{
Numerical investigation of the combined effects of curvature and normal stress on sheet metal formability
}

\author{
Mohamed Ben Bettaieb $^{1,2}$ \& Farid Abed-Meraim ${ }^{1,2}$ \& Xavier Lemoine ${ }^{1}$
}

\begin{abstract}
A number of parts and components involved in the automotive industry are made of thin bent sheets, which are subjected to out-of-plane compressive stresses in addition to traditional in-plane stresses. Unfortunately, the classical predictions based on the conventional concept of Forming Limit Diagram (FLD) are no longer relevant when the strain distribution is heterogonous over the sheet thickness. Therefore, this conventional FLD concept is not capable of accounting for the effect of out-of-plane stresses on the onset of localized necking. The aim of the present contribution is to propose an extension of the well-known Marciniak-Kuczynski approach to simultaneously account for the effect of curvature and normal stress on formability prediction. The new developed tool allows predicting the limit strains for the whole range of strain paths. The mechanical behavior of the studied sheets follows the rigid-plastic flow theory. Through numerical results, it is shown that both curvature and normal stress tend to increase the formability limit of the sheet metal.
\end{abstract}

Keywords Forming limit diagrams · Localized necking • Flow theory · Out-of-plane stress · Curvature · MarciniakKuczynskiapproach

\section{Introduction}

The study of ductility of metallic components and parts is an ambitious challenge in both academic and industrial applications. This ductility is often characterized by the classical concept of Forming Limit Diagram (FLD). This concept has been originally introduced by Keeler and Backofen [1], for representing the limit strains in the range of positive strain paths, and has been extended by Goodwin [2] to the whole range of strain paths. Due to the complexity of the experimental characterization of FLDs and its relatively high cost, a number of theoretical and / or numerical models have been developed in the literature. These models generally require the use of an instability criterion along with a constitutive law to describe the evolution of the mechanical state of the studied sheet. In the literature, a large number of models have been developed to numerically determine FLDs. Among these theoretical / numerical approaches, we can quote the work of Marciniak and Kuczynski [3], who coupled the initial imperfection approach with a rigid-plastic flow theory in order to predict the limit strains in the range of positive strain paths. Later, Hutchinson and Neale [4] extended the initial imperfection approach, originally developed in [3], to the range of negative strain paths. In the latter work, both the rigid-plastic flow theory and the deformation theory of plasticity were used to model the mechanical behavior of the studied sheet. In most of the traditional approaches proposed in the past, the predictions are generally based on three main assumptions: the homogeneity of the deformation through the thickness and in the plane of the sheet, the linearity of the strain paths, and the absence of out-of-plane stresses (a plane-stress state is assumed). In practice, however, these assumptions are seldom satisfied in most sheet metal forming processes. Indeed, in forming processes such as deep drawing or single point incremental forming, a punch or round tipped tool exerts on the 
sheet a force normal to its plane to deform it. In hydroforming processes, this force is exerted in the form of hydraulic pressure. Therefore, the effects of the magnitude of this normal stress and its distribution should be considered in the prediction of formability. Furthermore, the impact of curvature, created by the application of various forming tools, on formability is not taken into account, since homogeneous deformation through the sheet thickness is assumed in predictions based on the classical concept of FLDs. In the literature, few numerical studies have been carried out to investigate the abovementioned effects on formability:

- Effect of curvature on formability: It is not uncommon that metallic sheets used in industrial applications (automotive, aeronautic ...) undergo combination of bending and stretching deformations. Such modes of deformation may have a substantial impact on the formability of the studied parts. Several experimental studies revealed that bending tends to enhance the formability of metal sheets. In recent years, there have been a number of attempts to develop FLD prediction models that take into account bending effects. In this field, Shi and Gerdeen [5] integrated the effect of strain gradient and curvature in the prediction of FLDs for anisotropic materials. Sriram et al. [6] developed an empirical model to characterize fracture behavior during forming of advanced high strength steels under bending dominated conditions. He et al. [7] extended the initial imperfection approach developed in [3] to the case of combined stretching-bending loading. However, their work was restricted to the right-hand side of the FLD, where bending is along the major stretch direction. Two different constitutive laws were used in [7] to model the mechanical behavior of the sheet: the flow theory and the deformation theory of plasticity. The obtained numerical results suggested that bending process decreases sheet metal formability when the flow theory is used, whereas the opposite trend was observed with the deformation theory. Furthermore, the model developed in [7] was combined with finite element analysis in order to predict the forming limits of a sheet metal undergoing continuous bending-under-tension loading [8]. More recently, Safdarian [9] developed a new model for predicting FLDs of tailor welded blanks. In this model, the M-K approach developed in [4] has been enriched by taking into account the effect of bending strain on sheet metal formability. The flow theory of plasticity was used to describe the mechanical behavior of the studied sheet. Contrary to the approach developed in [6], the modified M-K model developed in [9] allows predicting the limit strains for the whole range of strain paths. However, this model presents some drawbacks and limitations. Indeed, in this model, the strain paths, both within the band and outside it, are assumed to remain linear during deformation. Consequently, the increment of strain components is replaced by total strain components in the constructive equations governing the associated modified M-K model. This assumption is not relevant in the band zone, where it is known that the strain path is constantly changing along deformation. Besides, the shear stresses have been withdrawn in the formulation of the equilibrium equations. This point represents the second limitation of the model. Indeed, the shear stresses cannot be neglected, especially when the band is not perpendicular to the major strain direction. In the present paper, a new modified M-K model is developed to address the above-mentioned issues. The main result of this paper is that the addition of bending effects tends to enhance sheet metal formability for the whole range of strain paths. This result confirms the numerical predictions reported in [8]. It must be noted that in all the previous studies dedicated to the investigation of curvature effects on the enhancement of formability, the plane-stress assumption has been adopted in the constitutive models used.

- Effect of normal stress on formability: Several classical localization criteria have been extended in the literature to take into account the effect of normal stress on formability. In this regard, one can quote Gotoh et al. [10] who extended both the Swift diffuse necking criterion [11] and the Hill localized necking criterion [12] to a 3D stress state, where the effects of normal stress on the onset of diffuse and localized necking have been accounted for. More recently, Allwood and Shouler [13] extended the initial imperfection approach to consider the effect of normal stress components on the prediction of limit strains. In the latter contribution, an isotropic rigid-plastic flow theory has been used to describe the mechanical behavior of the studied sheet. As a result of this investigation, a new generalized forming limit diagram has been proposed, which highlights the influence of normal stress components on the formability limits. The numerical approach developed in Allwood and Shouler [13] has been extended by Fatemi and Dariani [14] by taking into account the plastic anisotropy of the sheet via the use of the Hill'48 yield criterion. In both of these contributions, the strain and stress distributions have been assumed to be homogeneous through the thickness of the sheet during the deformation. In the current investigation, the $\mathrm{M}-\mathrm{K}$ extension that accounts for bending effects is further extended to take into account the effect of a heterogeneous distribution of compressive normal stresses over the thickness of the sheet. The magnitude of this stress distribution is assumed to be maximal in the inner surface of the sheet (which is assumed to be in contact with the tool) and zero in the outer surface of the sheet. To the authors' best knowledge, this is the first time the effects of curvature and normal stress on formability are simultaneously investigated within an extended $\mathrm{M}-\mathrm{K}$ model specifically designed to this purpose.

The remainder of the paper is organized as follows: 
- $\quad$ The equations governing the newly modified M-K extension will be detailed in the second section.

- In the third section, the numerical and algorithmic aspects relating to the model will be presented.

- Various numerical results obtained by application of the developed model will be presented and discussed in the fourth section.

\section{Notations}

The following notations and abbreviations are adopted in this paper:

\section{$-\quad \bullet^{\mathrm{T}}$ : transpose of tensor $\bullet$.}

- $\quad \bullet^{\mathrm{B}}$ : quantity $\bullet$ associated with the band.

- $\bullet^{\mathrm{H}}$ : quantity $\bullet$ associated with the zone located outside the band.

- $\quad \bullet_{\mathrm{ST}}$ : the stretching part of quantity $\bullet$.

- $\quad \bullet_{\mathrm{BE}}$ : the bending part of quantity $\bullet$.

- $\bullet(\mathrm{t})$ : value of quantity $\bullet$ at time $\mathrm{t}$.

- $\quad \operatorname{tr}(\bullet)$ : trace of tensor $\bullet$.

- $\bullet$ i: quantity $\bullet$ associated with the integration point i.

These notations can be combined. For instance, the stretching part of the strain tensor in the band is denoted by \&P.

\section{Governing equations}

\section{Mechanical behavior}

Elasticity is neglected in the subsequent constitutive equations. This assumption is justified, because strain localization occurs at relatively large strains. Moreover, the plastic flow is assumed to be isotropic and incompressible. The rigid-plastic flow theory is then used to model the mechanical behavior of the studied sheet metal. Hence, the strain rate tensor $\dot{\varepsilon}$ is derived by using the normality flow rule

$\dot{\varepsilon} 1 / 4 \dot{\lambda} \frac{\partial \sigma}{\partial \sigma} \frac{\mathrm{eq}}{;}$

where $\dot{\lambda}$ is the plastic multiplier (equal here to the von Mises equivalent strain rate $\varepsilon_{\mathrm{eq}}$ ), $\sigma$ is the Cauchy stress tensor, and $\sigma_{\text {eq }}$ is the von Mises equivalent stress. By using the definition of $\sigma_{\mathrm{eq}}$, the rigid-plastic constitutive law can be derived from Eq. (1)

$\dot{\varepsilon} 1 / 4 \frac{3 \dot{\varepsilon}_{\mathrm{eq}}}{2 \sigma_{\mathrm{eq}}} \mathrm{S} \Leftrightarrow \mathrm{S} 1 / 4 \frac{2 \sigma_{\mathrm{eq}}}{3 \dot{\varepsilon}_{\mathrm{eq}}} \dot{\varepsilon} ;$

where $S$ is the deviatoric part of $\sigma$. These two tensors are linked by the following relation: $\sigma 1 / 4 \mathrm{~S} \mathrm{p} \mathrm{pI}_{2}$;

ð3Р

where $\mathrm{p}$ is the hydrostatic pressure equal to $1 / 3 \operatorname{tr}(\sigma)$ and $\mathrm{I}_{2}$ is the second-order identity tensor.

The equivalent stress $\sigma_{\text {eq }}$ is related to the equivalent strain $\varepsilon_{\text {eq }}$ by the Hollomon isotropic hardening law

$\sigma_{\text {eq }} 1 / 4 K \varepsilon_{e}^{n}$;

where $\mathrm{K}$ and $\mathrm{n}$ are two material hardening parameters.

By combining Eqs. (2) and (4), one obtains

$S{ }_{1 / 4}^{-} \underset{3 \dot{\varepsilon}_{\text {eq }}}{-2 K \varepsilon^{n}}$

ð5P

Modified M-K approach

Assumptions related to the Bending-Stretching process

The sheet is assumed to be initially flat, with $\mathrm{X}$ and $\mathrm{Y}$ the two in-plane principal directions and $\mathrm{Z}$ the through-thickness direction, as illustrated in Fig. 1. It should be understood that the $\mathrm{X}-\mathrm{Y}-\mathrm{Z}$ coordinate system represents a material coordinate frame, which will rotate as material deforms. The following assumptions and choices are made in the development of this modified M-K approach:

- The sheet is first subjected to a bending moment $\mathrm{M}$ applied in Y-direction, resulting in the sheet being curved along X-direction, with a uniform curvature R. Hence, there is a single curvature along $\mathrm{X}$-direction, while the sheet remains straight along the Y-direction (see Fig. 1).

- The neutral axis is assumed to remain located at the midlayer of the sheet metal during deformation.

- $\quad$ The studied sheet is assumed to be wide enough (along the Y-direction) relative to its thickness. Consequently, the strain component $\varepsilon_{\mathrm{yy}}$ can be neglected during the bending process.

- $\quad$ The sheet is initially submitted to a heterogeneous distribution of out-of-plane stress through its thickness. This out-of-plane stress distribution is assumed to take a constant value during the deformation for each surface locat-

ed at a given distance from the mid-layer of the sheet.

- After the bending stage, an axial major strain along $\mathrm{X}$ and a minor strain along $\mathrm{Y}$ are then applied to the sheet under a constant strain-path ratio $\rho$, while the bending radius is kept constant equal to $\mathrm{R}$.

Modified M-K equations

The $\mathrm{M}-\mathrm{K}$ approach is based on the assumption of the preexistence of an initial geometric imperfection in the form of a 


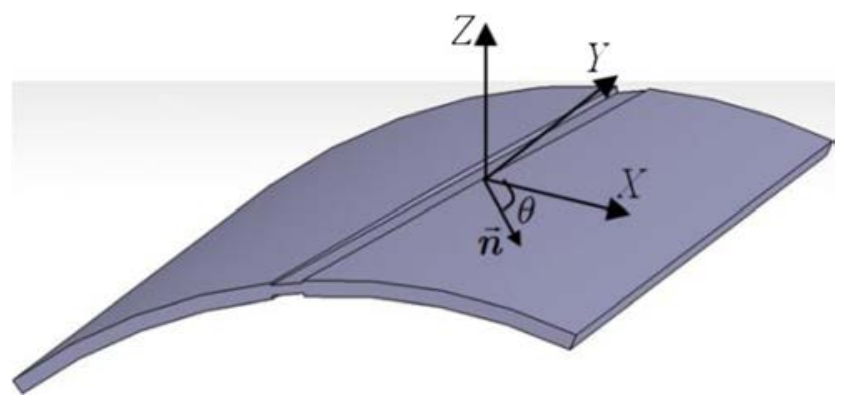

Fig. 1 Initial imperfection approach for the sheet metal

band across the thickness of the sheet, as illustrated in Fig. 1. This band is defined by its normal unit vector $n$. The angle

between vector $n$ and the major strain direction $X$ is denoted $\theta$. The initial thickness of the band (resp. zone outside the band) is equal to $\mathrm{h}_{0}^{\mathrm{B}}$ (resp. $\mathrm{h}_{0}^{\mathrm{H}}$ ).

The sheet illustrated in Fig. 1 is subjected to biaxial stretching in the $\mathrm{X}$ and $\mathrm{Y}$ directions. Additionally, a non-zero principal stress $\sigma_{z z}$ acts in the out-of-plane direction (i.e., in the Z-direction). The distribution of this stress component is assumed to be homogeneous through the plane of the sheet (i.e., $\sigma_{z z}$ is independent of $x$ and y coordinates), but heterogeneous over the thickness of the sheet. The value of $\sigma_{z z}$ is taken constant for each surface located at a given distance from the mid-layer of the sheet. For example, $\sigma_{\mathrm{zz}}$ is equal to $-\alpha$ at the inner surface of the sheet (where $\alpha$ is a non-negative pressure, constant during the deformation), while it is equal to 0 at the outer surface. Also, $\sigma_{\mathrm{zz}}$ is assumed to evolve linearly, as a function of the z coordinate between the two external surfaces
of the sheet. The distribution and the magnitude of $\mathrm{o}_{z z}$ are assumed to be the same both in the homogeneous zone and

\section{Bn the band As a conseguence of the assumptions made in}

section $^{\wedge}$ and the spatial distribution of $\sigma_{\mathrm{zz}}$, it can be shown

that the different mechanical variables (strain, stress...) are independent of the $\mathrm{x}$ and $\mathrm{y}$ coordinates, both inside and outside the band. These mechanical variables only depend on the $\mathrm{z}$ coordinate. Under the incompressibility condition, it is more convenient to rewrite in what follows the governing equations in the form of an in-plane formulation (i.e., in the plane (X Y)).

The total strain $\varepsilon$ is additively decomposed into a bending part $\varepsilon_{\mathrm{BE}}$ and a stretching part $\varepsilon_{\mathrm{ST}}$

$\varepsilon 1 / 4 \varepsilon_{\mathrm{BE}} p \varepsilon_{\mathrm{ST}}:$

The above decomposition is valid both inside and outside the band.

Also, the bending part $\varepsilon_{\mathrm{BE}}$ of the deformation tensor has
The stretching part $\dot{\varepsilon}_{\mathrm{ST}}^{\mathrm{H}}$ is assumed to be uniform over the thickness of the sheet and is given by the following expression:

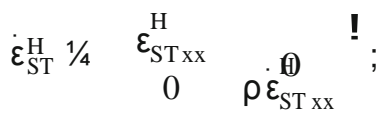

where the strain-path ratio $\rho$ is varied in the range $-1 / 2 \leq \rho \leq 1$ to span the complete FLD.

The stretching part of the strain rate in the band $\dot{\varepsilon}_{\mathrm{ST}}^{\mathrm{B}}$ is related to that outside the band $\dot{\varepsilon}_{\mathrm{ST}}^{\mathrm{H}}$ by the following kinematic compatibility condition:

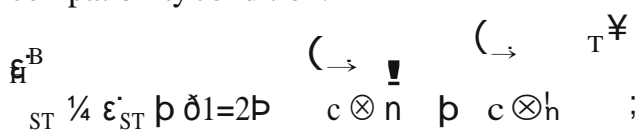

ð9P

where $\stackrel{\text { ! }}{\mathrm{c}}$ is the jump vector. Vectors $\stackrel{!}{\mathrm{c}}$ and $\mathrm{n}$ are assumed to be uniform over the thickness of the sheet.

The evolution of the band orientation $\theta$ is given by the following relation:

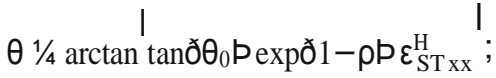

where $\theta_{0}$ is the initial value of the band orientation.

The global equilibrium equation across the band can be expressed as follows:

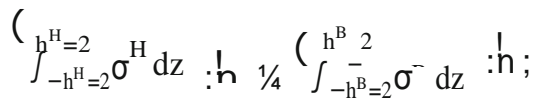

ð11P

where $h^{\mathrm{H}}$ and $\mathrm{h}^{\mathrm{B}}$ are the current thicknesses of the sheet outside and in the band zone respectively, These thicknesses are
expressed as functions of their initial values $h^{4}$ the components of $\dot{\varepsilon}^{\mathrm{H}} \cdot \mathrm{B} \quad 0$ and $\mathrm{h}_{0}$ and of

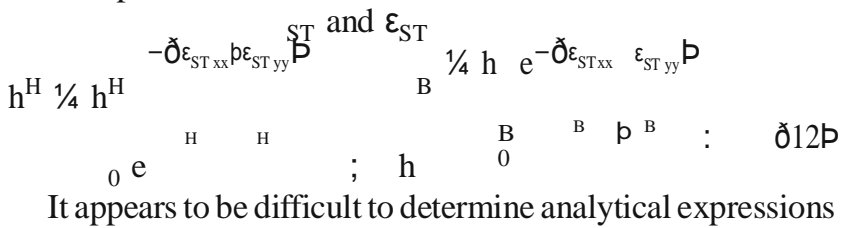
providing exact values for the two integrals introduced in Eq. (11). Therefore, these integrals are rather numerically approximated by the trapezoidal method, after a geometric discretization of the sheet thickness is performed

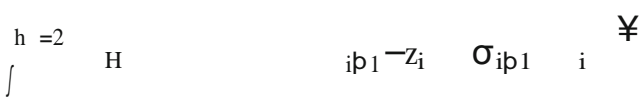

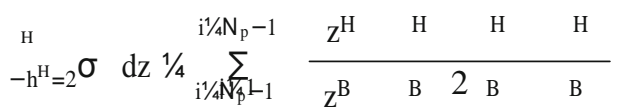

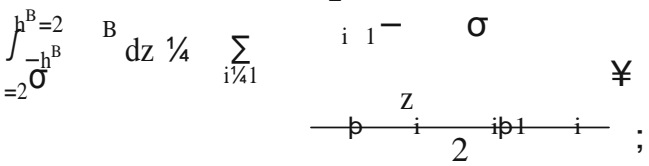

the same expression in both zones 
where $\mathrm{N}_{\mathrm{p}}$ is the number of integration points used in the numerical quadrature, which is asşumed, for convenience, to be the $\varepsilon_{\mathrm{BE}}{ }^{1 / 4} \begin{array}{cc}\text { Lnð1 } \mathrm{p}_{\mathrm{z}}=\mathrm{RP} & 0 \\ 0 & 0\end{array}$ : same for both zones. In Eq. (12), $z_{i}^{\mathrm{H}}$ (resp. $z_{i}^{\mathrm{B}}$ ) represents the current position of the $i^{\text {th }}$ integration point through the thickness in the zone outside the band (resp. in the band). Hence, $\mathrm{z}_{1}, \mathrm{Z}_{\mathrm{N}_{\mathrm{p}}}$, $\mathrm{Z}^{\mathrm{B}} \quad \mathrm{B} \quad \mathrm{H} \quad \mathrm{H} \quad \mathrm{B} \quad \mathrm{B}$ ${ }_{1}, \mathrm{z}_{\mathrm{N}_{\mathrm{p}}}$ are equal to $-\mathrm{h} / 2, \mathrm{~h} / 2,-\mathrm{h} / 2, \mathrm{~h} / 2$, respectively. 
Furthermore, $z_{i}^{\mathrm{H}}$ (resp. $\mathrm{z}_{i}^{\mathrm{B}}$ ) is related to its initial value $\mathrm{z}_{\mathrm{i} 0}^{\mathrm{H}}$ (resp. $z_{i 0}^{\mathrm{B}}$ ) by

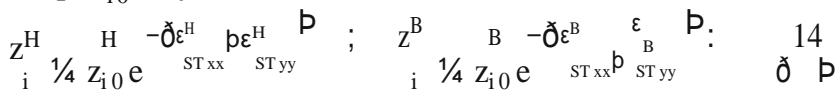

\section{Algorithmic aspects}

\section{Bending process}

Before the stretching loading is applied, the sheet metal is first bent by applying a bending moment $\mathrm{M}$. During this bending stage, the curvature radius decreases from $+\infty$ (which corresponds to a flat sheet) to a finite value $\mathrm{R}$. This bending is moderate compared to stretching (typically the ratio $\mathrm{h}_{0}^{\mathrm{H}}=\mathrm{R}$ does not exceed 0.2). Therefore, it is legitimate to consider the distance $\mathrm{z}$ of any integration point to the neutral axis as a constant value, which is taken equal to its initial value $\mathrm{z}_{0}$. At the end of this bending process, the equivalent strains corresponding to the different points located inside and outside the band are computed

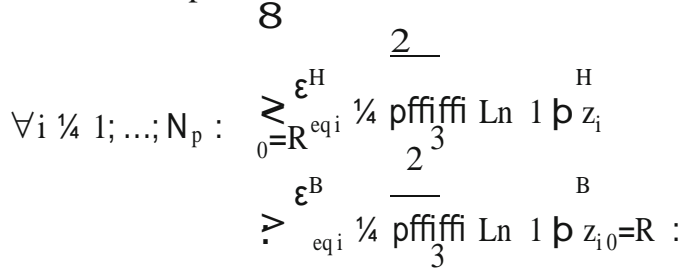

Simultaneously to this bending loading, a normal stress, which is constant during the loading and heterogeneous, is applied on both the safe zone and the band. As previously discussed, this normal stress is linearly distributed through the thickness of the sheet, as defined by the following relations:

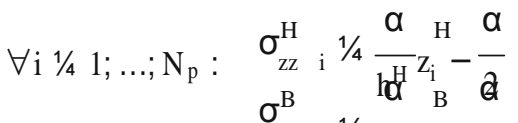

$$
\begin{aligned}
& \sigma^{\mathrm{B}} \mathrm{i}^{1 / 4} \frac{}{h^{\mathrm{B}} z_{i}}-\frac{1}{2} \text { : }
\end{aligned}
$$

\section{Algorithm for the FLD prediction}

The general algorithm developed for the FLD prediction is based on the following three nested loops:

- For each strain-path ratio $\rho$ ranging from $-1 / 2$ to 1 (with increments $\Delta \rho$ of 0.1 ).

- For each initial band orientation $\theta_{0}$, spanning the admissible range of inclination angles (i.e., between $0^{\circ}$ and $90^{\circ}$ ), at user-defined intervals (here, we take intervals of $1^{\circ}$ ).

For each time interval $\left[\mathrm{t}_{0}, \mathrm{t}_{0}+\Delta \mathrm{t}\right]$, apply the implicit incremental algorithm described in BIncremental integration of the governing equations section ${ }^{\wedge}$ to integrate the governing equations detailed in BGoverning equations section^. The application of this incremental
integration scheme is stopped when the following criterl-

on is satisfied:

$\Delta \varepsilon_{\mathrm{STzz}}^{\mathrm{B}}=\Delta \varepsilon_{\mathrm{STzz}}^{\mathrm{H}} \geq 10$ :

The strain component $\varepsilon_{\mathrm{x}}^{\mathrm{H}}$, thus obtained once the criterion (17) is satisfied, is considered as being the critical strain $\varepsilon_{\mathrm{x}}^{*}$ corresponding to the initial band inclination $\theta_{0}$ and strain-path ratio $\rho$. The factor 10 in Eq. (17) is rather arbitrary and any other relatively large positive value can be used without loss of accuracy. Indeed, the impact of this value on the predicted critical strain is minimal, since the absolute value of the band thickness strain rate $\dot{\varepsilon}_{\mathrm{STzz}}^{\mathrm{B}}$ increases very rapidly towards infinity at incipient necking.

The smallest critical strain $\varepsilon_{\mathrm{x}}^{*}$, over all initial angles $\theta_{0}$, and the corresponding current angle, define the necking limit strain $\varepsilon_{x}^{L}$ and the necking band orientation, respectively, for the current strain-path ratio $\rho$.

The above-developed algorithm and the associated algorithmic aspects have been implemented in a standalone code developed using the multi-paradigm numerical computing environment Mathematica. This implementation is carried out independently of any FE code. The input data for this code are all parameters related to the material (isotropic hardening), geometry (thickness of the sheet and curvature radius), mechanical state (amount and distribution of the normal stress) and numerical choices (number and position of the different integration points). On the other hand, the output results are the limit strain $\varepsilon^{L}$ and the necking band orientation corresponding to the strain-path ratio $\rho$.

\section{Incremental integration of the governing equations}

The main purpose of this incremental algorithm is to integrate the equations governing the modified $\mathrm{M}-\mathrm{K}$ approach over a typical time increment $\left[\mathrm{t}_{0}, \mathrm{t}_{0}+\Delta \mathrm{t}\right]$. In this aim, we assume that, at each integration point located in the zone outside the band or in the band, the following quantities are known at time $\mathrm{t}_{0}$ :

- $\quad$ The coordinate through the thickness direction z (see Eq. 14).

- $\quad$ The equivalent strain $\varepsilon_{\text {eq }}$ (see Eq. 15)

- The normal stress $\sigma_{\mathrm{zz}}$ (see Eq. 16).

In order to simplify notations, the argument $t_{0}+\Delta t$ will be omitted hereinafter, with the implied understanding that the 
corresponding quantity is evaluated at $t_{0}+\Delta t$, unless otherwise indicated.

The increment $\Delta \varepsilon_{\text {ST zz }}^{\mathrm{B}}$ is chosen as loading parameter over $\left[\mathrm{t}_{0}, \mathrm{t}_{0}+\Delta \mathrm{t}\right]$. It is typically set to -0.001 .

The increment $\Delta \varepsilon_{\mathrm{ST}}^{\mathrm{H}}$ is derived from the integration of Eq. (8):

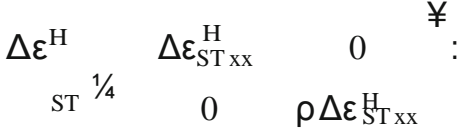

On the other hand, the components of the increment $\Delta \varepsilon_{\text {ST }}^{\mathrm{B}}$ are derived from the integration of Eq. (9)

$\Delta \varepsilon_{\mathrm{STXx}}^{\mathrm{B}} \quad 1 / 4 \Delta \varepsilon_{\mathrm{STxx}}^{\mathrm{H}} \mathrm{p} \Delta \mathrm{c}_{1} \mathrm{n}_{1}$

$\Delta \varepsilon_{\text {STxy }}^{\mathrm{B}} 1 / 4 \Delta \varepsilon_{\text {STyx }}^{\mathrm{B}} 1 / 4$ ð1=2p $\partial \Delta \mathrm{c}_{1} \quad \mathrm{n}_{2} \mathrm{p} \Delta \mathrm{c}_{2} \mathrm{n}_{1} \mathrm{P}$

$\Delta \varepsilon_{\text {STyy }}^{\mathrm{B}}{ }_{\text {ST xx }}^{1 / 4} \rho \Delta \varepsilon^{\mathrm{H}}$ p $\Delta \mathrm{c}_{2} \mathrm{n}_{2}$ :

It is noteworthy that Eq. (19) has been derived from Eq. (9) by implicitly assuming that, all along the time increment, the components of vector $n$ keep their initial values at $t_{0}$.

Furthermore, Eq. (19) in conjunction with the incompressibility condition leads to the following relation:

ð1 p pp $\Delta \varepsilon_{\text {ST xx }}^{\mathrm{H}} \mathrm{p} \Delta \mathrm{c}_{1} \quad \mathrm{n}_{1} \mathrm{p} \Delta \mathrm{c}_{2} \quad \mathrm{n}_{2} 1 / 4-\Delta \varepsilon_{\text {STzz }}^{\mathrm{B}}: \quad$ ð20

In the band, the position of the different integration points at $\mathrm{t}_{0}+\Delta \mathrm{t}$ can be easily expressed in terms of $\Delta \varepsilon_{\mathrm{ST}}^{\mathrm{B}} \quad$ zz

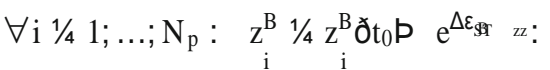

By using Eqs. (7) and (21), the increment of $\varepsilon_{\mathrm{BE}}^{\mathrm{B}}$ over [ $\mathrm{t}_{0}$, $t_{0}+\Delta t$, for the different integration points within the band, can be expressed as

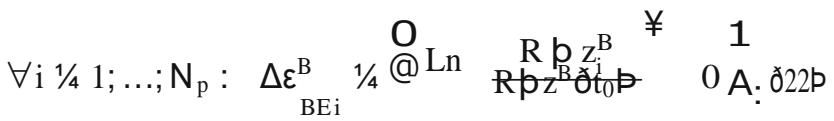

$$
\begin{aligned}
& 0^{\mathrm{i}} \quad 0
\end{aligned}
$$

The position at $t_{0}+\Delta t$ of the integration points located outside the band can be determined by the following equation:

$\forall \mathrm{i} 1 / 41 ; \ldots ; \mathrm{N}_{\mathrm{p}}: \quad \mathrm{z}_{\mathrm{i}}^{\mathrm{H}} \frac{1 / 4}{\mathrm{i}} \mathrm{z}_{\mathrm{i}}^{\mathrm{H} \mathrm{t}_{0}} \mathrm{Pe}^{\Delta \varepsilon_{\mathrm{Mzz}}} ;$

where $\Delta \varepsilon_{\mathrm{STzz}}^{\mathrm{H}}$ is determined by applying the incompressibility condition

$\Delta \varepsilon_{\mathrm{STzz}}^{\mathrm{H}} 1 / 4-\partial 1 \mathrm{p} \rho \mathrm{DP} \Delta \varepsilon_{\mathrm{ST} \mathrm{xx}}^{\mathrm{H}}:$

The increment of $\varepsilon_{\mathrm{BE}}^{\mathrm{H}}$ over [ $\mathrm{t}_{0}, \mathrm{t}_{0}+\Delta \mathrm{t}$ ] for the different integration points located outside the band is determined by a relation very similar to Eq. (22)

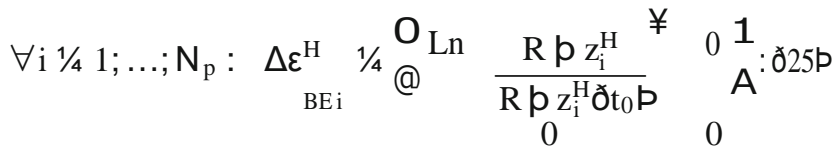

The increment over $\left[\mathrm{t}_{0}, \mathrm{t}_{0}+\Delta \mathrm{t}\right]$ of the strain tensors $\varepsilon^{\mathrm{H}}$ and $\varepsilon^{\mathrm{B}}$ at the different integration points located outside and in the band zone is finally determined as follows:

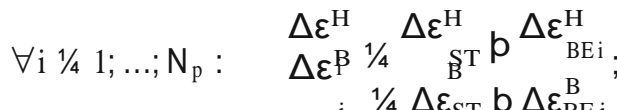

ð26P

where the expressions of $\Delta \varepsilon^{\mathrm{H}}, \Delta \varepsilon^{\mathrm{H}}, \Delta \varepsilon^{\mathrm{B}}$, and $\Delta \varepsilon^{\mathrm{B}}$ are

$$
\text { ST BE i ST BE i }
$$

given by Eqs. (18), (19), (22), and (25), respectively. Accordingly, for a given strain-path ratio $\rho$, the strain increments $\Delta \varepsilon^{\mathrm{H}}$ and $\Delta \varepsilon^{\mathrm{B}}$ are functions of three scalar unknowns: $\Delta \varepsilon_{\mathrm{ST} \mathrm{xx}}^{\mathrm{H}}, \Delta \mathrm{C}_{1}$ and $\Delta \mathrm{c}_{2}$.

The expression of the deviatoric stress $\mathrm{S}$ at the different integration points inside and outside the band is derived from Eq. (5)

$\mathrm{S} 1 / 4 \frac{2 \mathrm{~K} \varepsilon_{\mathrm{e}}^{\mathrm{n}}}{3 \Delta \varepsilon_{\mathrm{eq}}} \Delta \varepsilon$

The Cauchy stress tensor $\sigma$ can be expressed as follows:

$$
\begin{aligned}
& { }_{\sigma_{\mathrm{xx}}}^{0} \quad 0^{1} \\
& \begin{array}{cccc}
\sigma^{1 / 4} & 0 & \sigma_{y y} & 0 \\
& 0 & 0 & \sigma_{z z}
\end{array}
\end{aligned}
$$

Tensor $\sigma$ is related to its deviatoric part $\mathrm{S}$ by

$S \frac{1}{1 / 4} \sigma-ð 1=3 P$ trð $\sigma \mathrm{P} \mathrm{I}_{2}$ :

Therefore, the components of tensor $\mathrm{S}$ are given by the following relations:

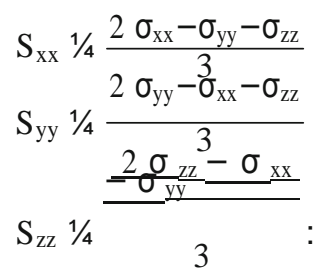

ð30p

By inverting the above relations, one can obtain the expressions of $\sigma_{\mathrm{xx}}$ and $\sigma_{\mathrm{yy}}$ as functions of $\mathrm{S}_{\mathrm{xx}}, \mathrm{S}_{\mathrm{yy}}$ and $\sigma_{\mathrm{zz}}$

$\sigma_{x x} 1 / 42 S_{x x} p S_{y y} p \sigma_{z z} \quad ; \quad \sigma_{y y} 1 / 42 S_{y y} p S_{x x} p \sigma_{z z}:$ z31p

The distribution of the normal stress $\sigma_{\mathrm{zz}}$ over the thickness of the sheet is given by Eq. (16).

Combining Eqs. (3), (27) and (31), one can easily derive the expression of the Cauchy stress tensor $\sigma$ at the different integration points over the thickness of the sheet.

Once determined, the expression for $\sigma$ is then inserted in the approximation (13), which in turn is inserted in the equilibrium Eq. (11). Analyzing the previous developments, Eq. (11) may be regarded as a system of two equations with three scalar unknowns: $\Delta \varepsilon_{\mathrm{STxx}}^{\mathrm{H}}, \Delta \mathrm{c}_{1}$ and $\Delta \mathrm{c}_{2}$. By considering Eq. (11) along with Eq. (20), a system of three scalar equations with the three above unknowns is obtained. This system 
should be solved iteratively by using the Newton-Raphson method in order to determine the above-mentioned scalar unknowns. Ultimately, the determination of these unknowns allows computing the different quantities at $\mathrm{t}_{0}+\Delta \mathrm{t}$ : namely, the distance of the different integration points to the neutral axis of the sheet, the distribution of the equivalent strain through the thickness in both zones, and the band orientation. In particular, the values of the strain components $\Delta \varepsilon_{\text {STzz }}^{\mathrm{H}}$ and $\varepsilon_{\text {ST Xx }}^{\mathrm{H}}$ (the latter being equal to $\varepsilon_{\text {ST xx }}^{\mathrm{H}} \partial_{0} \mathrm{Pp} \Delta \varepsilon_{\mathrm{ST} \mathrm{xx}}^{\mathrm{H}}$ ) are of special interest; the former being required for the application of the necking criterion (17), while the latter is needed in the algorithm of BAlgorithm for the FLD prediction section^.

\section{Prediction results}

The current section is divided into four subsections:

- The material and geometric data are briefly presented in the first subsection.

- The effect of curvature on the enhancement of formability is demonstrated in the second subsection through several numerical simulations (under plane-stress assumption).

- The third subsection is dedicated to the investigation of the effect of normal stress on the formability of a flat sheet.

- The combined effects of curvature and normal stress on formability are depicted in the fourth subsection.

Material and geometric data

A DP600 sheet steel with the hardening parameters $\mathrm{K}=$ $945 \mathrm{MPa}$ and $\mathrm{n}=0.16$ is used in the following simulations The initial thicknesses $h^{12}$ and $h^{3}$ are set to $1 \mathrm{~mm}$ and $0.99 \mathrm{~mm}$, respectively. The number of integration points $\mathrm{N}_{\mathrm{p}}$ is taken equal to 11 .

\section{Effect of curvature}

Figure 2 shows the evolution of the ratio $\Delta \varepsilon_{\mathrm{ST} \mathrm{zz}}^{\mathrm{B}}=\Delta \varepsilon_{\mathrm{ST} \text { zz }}^{\mathrm{H}}$ as a function of $\varepsilon_{\text {ST xx }}^{\mathrm{H}}$ for both cases of plane strain tensile state $(\rho=0)$ and equibiaxial tensile state $(\rho=1)$. In this figure, three bending radii are considered: $\mathrm{R}=3 \mathrm{~mm} ; \mathrm{R}=10 \mathrm{~mm}$; $\mathrm{R}=$ $100 \mathrm{~mm}$. The results corresponding to a classical flat sheet are also included in this figure. The occurrence of strain localization is predicted when the ratio $\Delta \varepsilon_{\mathrm{STzz}}^{\mathrm{B}}=\Delta \varepsilon_{\mathrm{S}}^{\mathrm{H}} \mathrm{Tzz}$ exceeds 10 , as stated by criterion (17). It is clear from this figure that the value of $\varepsilon_{\text {ST xx }}^{\mathrm{H}}$ at the onset of strain localization increases with the bending radius $\mathrm{R}$. For large values of the bending radius $\mathrm{R}$ (e.g., more than $100 \mathrm{~mm}$ ), bending effect is very limited. In such conditions, the prediction with high bending a

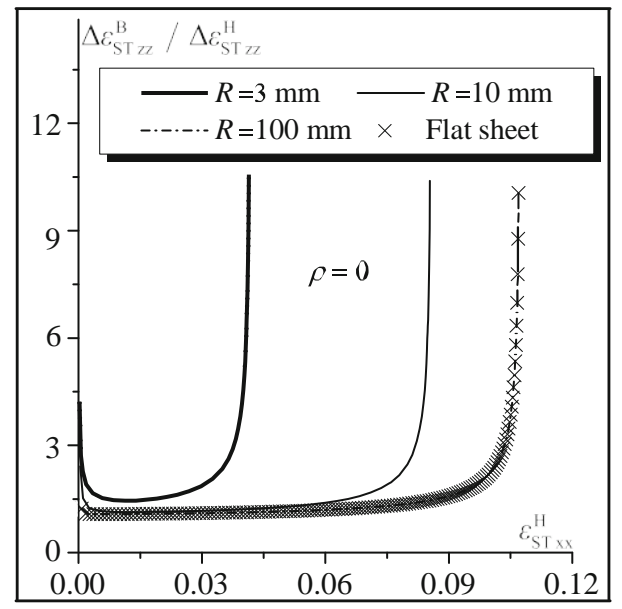

b

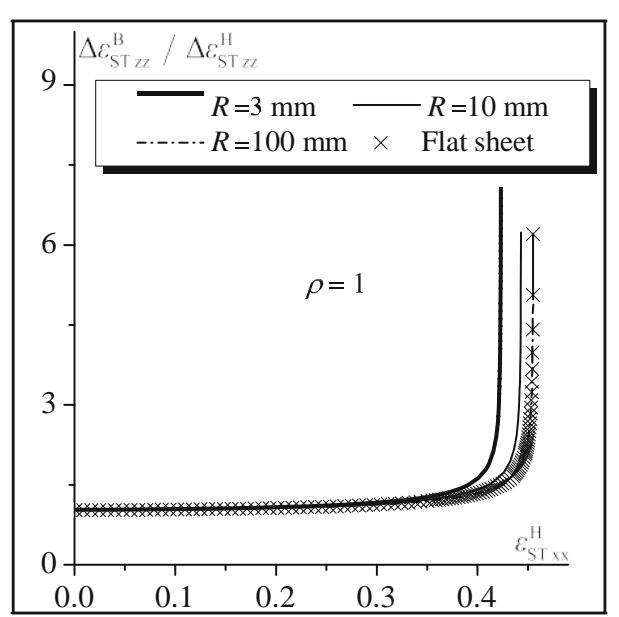

Fig. 2 Illustration of the onset of localized necking: evolution of the ratio $\Delta \varepsilon^{\mathrm{B}} \underset{\text { STzz }}{=}=\Delta \varepsilon_{\mathrm{ST} z z}^{\mathrm{H}}$ as a function of $\varepsilon_{\text {ST Xx }}^{\mathrm{H}}$ : (a) plane strain tensile state $(\rho=$
$0)$; (b) equibiaxial tensile state $(\rho=1)$

radius is almost the same as that corresponding to a flat sheet, as observed in Fig. 2.

Figure 3 depicts the evolution of the ratio $\Delta \varepsilon_{\mathrm{STzz}}^{\mathrm{B}}=\Delta \varepsilon_{\mathrm{STzz}}^{\mathrm{H}}$ as a function of $\varepsilon_{\mathrm{x}}^{\mathrm{H}}$ (the major deformation of the outer surface of the sheet) for both cases of plane strain tensile state $(\rho=0)$ and equibiaxial tensile state $(\rho=1)$. It must be noted that the strain component $\varepsilon_{x}^{\mathrm{H}}$ is the strain that is actually measurable during the deformation. It is clearly shown from this figure that the limit strain $\varepsilon_{\mathrm{x}}^{\mathrm{H}}$ decreases with the bending radius. This means that bending tends to retard the occurrence of localized necking and, thus, to enhance formability, especially when the bending radius is small. The difference in the trends between Figs. 2 and 3 is due to the contribution of the bending part to the strain measure: only the stretching contribution $\varepsilon_{\mathrm{S}_{\mathrm{xx}}}^{\mathrm{H}}$ has been used in Fig. 2, while the total strain component $\varepsilon_{\mathrm{x}}^{\mathrm{H}}$ is used in Fig. 3. As the bending strain component (which is equal to the difference between $\varepsilon_{\text {xx }}^{\mathrm{H}}$ and $\varepsilon^{\mathrm{H}}{ }_{\text {ST xx }}$ ) significantly decreases with the bending radius (see Eq. 25), this explains 
a

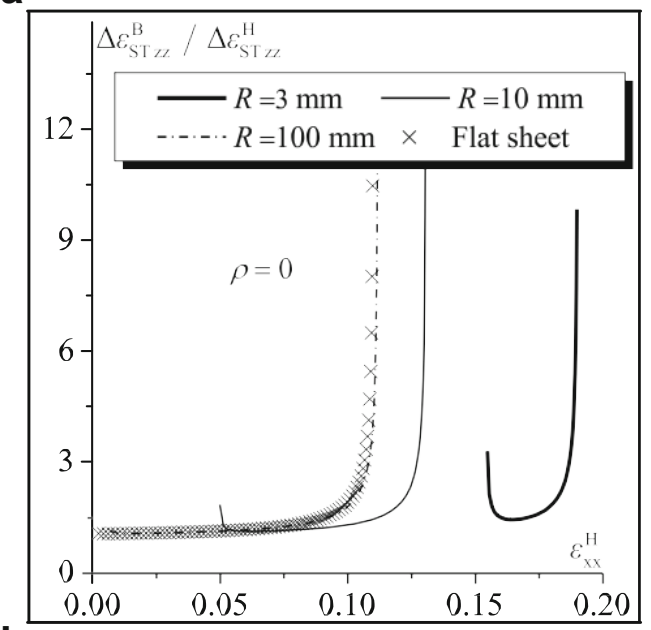

b

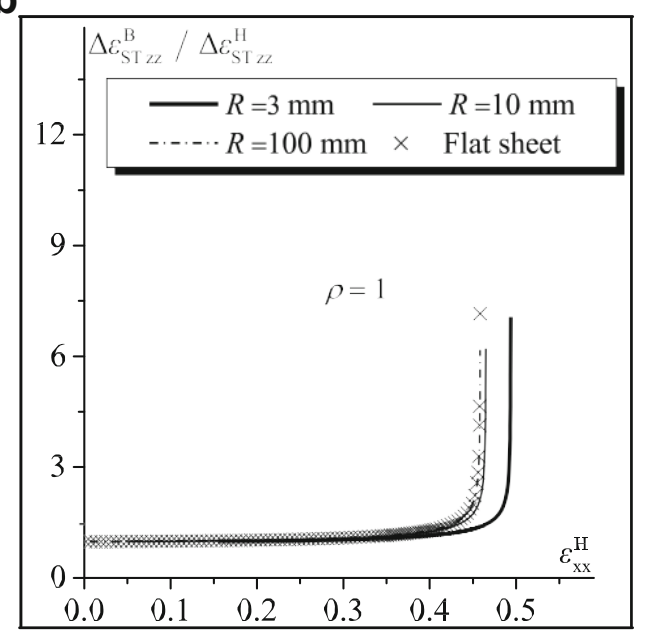

Fig. 3 Illustration of the onset of localized necking: evolution of the ratio $\Delta \varepsilon_{\mathrm{ST} \mathrm{zz}}^{\mathrm{B}}=\Delta \varepsilon_{\mathrm{ST} \mathrm{zz}}^{\mathrm{H}}$ as a function of $\varepsilon_{\mathrm{xx}}^{\mathrm{H}}$ : (a) plane strain tensile state $(\rho=0)$; (b) equibiaxial tensile state $(\rho=1)$

the opposite trends observed between Fig. 2 and Fig. 3. It must be noted that the evolution of the ratio $\Delta \varepsilon_{\text {STzz }}^{\mathrm{B}}=\Delta \varepsilon_{\mathrm{ST} \text { zz }}^{\mathrm{H}}$ is presented in Fig. 3 during the stretching phase only (i.e., the bending stage is not shown in this figure). This fact explains why the initial value of $\varepsilon_{\mathrm{x}}^{\mathrm{H}}$ (which correspond to the onset of the stretching phase) is not always equal to zero (especially for small to moderate bending radii, see Fig. 3 (a)).

To further investigate the effect of curvature on the onset of strain localization, the stretching limit strain $\varepsilon_{\text {ST xx }}^{\mathrm{L}}$ (the stretching strain determined at the onset of plastic strain localization) and the total limit strain $\varepsilon_{x}^{\mathrm{L}}$ (the sum of the stretching strain and the bending strain, as predicted at the beginning of localized necking) are plotted as functions of the inverse of the bending radius R in Fig. 4 (a) and Fig. 4 (b), respectively. In both figures, four representative strain paths are considered: $\rho=-0.5 ; \rho=0 ; \rho=0.5$; and $\rho=1$. The results of Fig. 4 (a) (resp. Fig. 4 (b)) confirm the observations displayed in Fig. 2 (resp. Fig. 3).
The effect of bending radius on the evolution of the stretching critical strain $\varepsilon_{\mathrm{ST} \text { xx }}^{*}$, as a function of the initial band orientation $\theta_{0}$, is investigated in Fig. 5 for the case of uniaxial tensile strain path. Note that the necking band is not perpendicular to the major strain direction, whatever the value of the bending radius $\mathrm{R}$. Indeed, the stretching critical strain $\varepsilon_{\mathrm{ST} \mathrm{xx}}^{*}$ decreases with the initial band orientation $\theta_{0}$ until reaching its lowest value, which corresponds to the stretching limit strain हाt $\mathrm{xx}$, and then it increases afterwards. This result is common to all negative strain paths, as demonstrated in Fig. 6 . However, for positive strain paths, the necking band is perpendicular to the major strain direction. These observations are classical for flat sheets when bending effects are not considered. Note that the effect of bending radius on the necking band orientation $\theta$ is not very significant, as shown in Fig. 6.

The results presented in Fig. 5 for the particular case of uniaxiale tensile strain path are extended in Fig. 7 to the whole

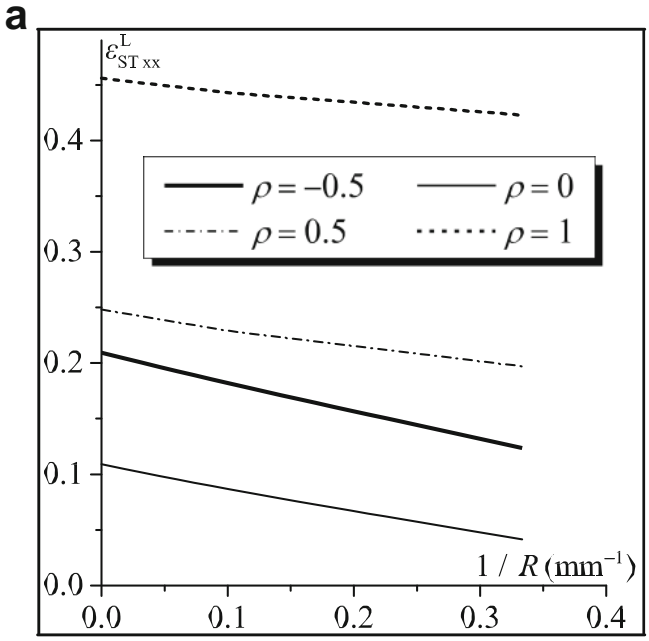

b

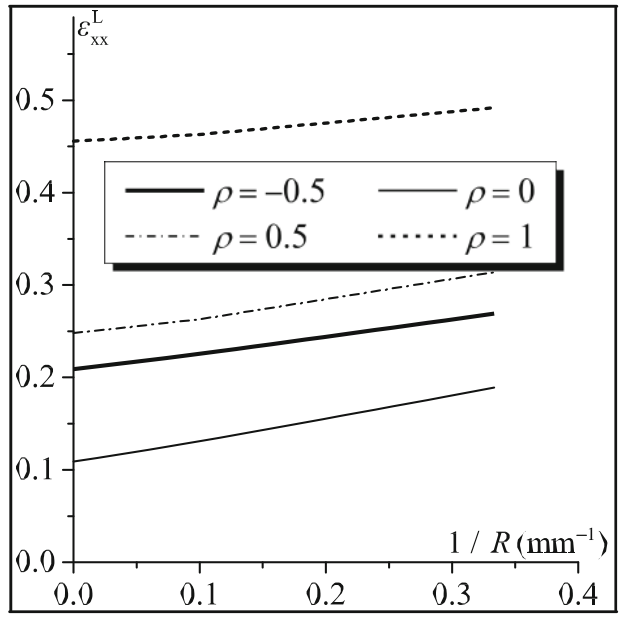

Fig. 4 Effect of curvature on the onset of localized necking: (a) Evolution of the stretching limit strain $\varepsilon_{\mathrm{ST} x \mathrm{Lx}}^{\mathrm{L}}$ as a function of the ratio 1/R; (b) Evolution of the total limit strain $\varepsilon_{S}^{\mathrm{L}}$ as a function of the ratio 1/R 


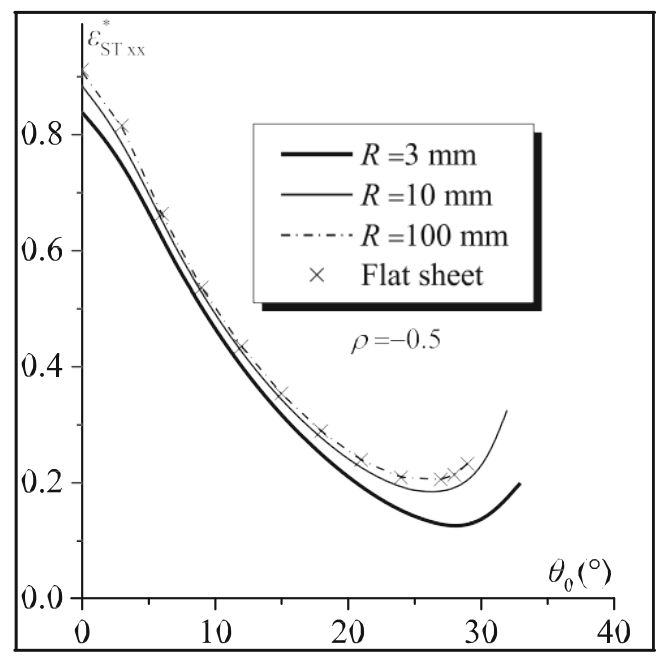

Fig. 5 Effect of the bending radius on the evolution of the stretching critical strain $\xi_{\text {TT }}^{*}$ as a function of the initial band orientation $\theta_{0}$ for uniaxial tensile strain path

range of strain paths, where the effect of bending radius on the location and shape of FLDs is analyzed. In Fig. 7 (a), the major stretching limit strain (simply denoted $\varepsilon_{\text {ST xx }}$ ) is plotted

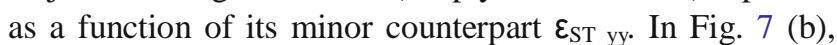
however, the major total limit strain at the outer surface of the sheet (simply denoted $\varepsilon_{\mathrm{xx}}$ ) is plotted as a function of its minor counterpart $\varepsilon_{y y}$. It should be noted that, as bending is not considered in the y-direction, $\varepsilon_{\text {ST yy }}$ is identically equal to $\varepsilon_{\mathrm{yy}}$ for the different strain paths. It appears from Fig. 7 (b) that the bending effect is more significant in the left-hand side of the FLD than in its right-hand side. The predictions reported in this figure confirm once again that the classical curvature has a beneficial effect on the formability of thin sheets. By comparing the results displayed in Fig. 7 (a) and Fig. 7 (b), one can easily observe that the two FLD representations (i.e., that

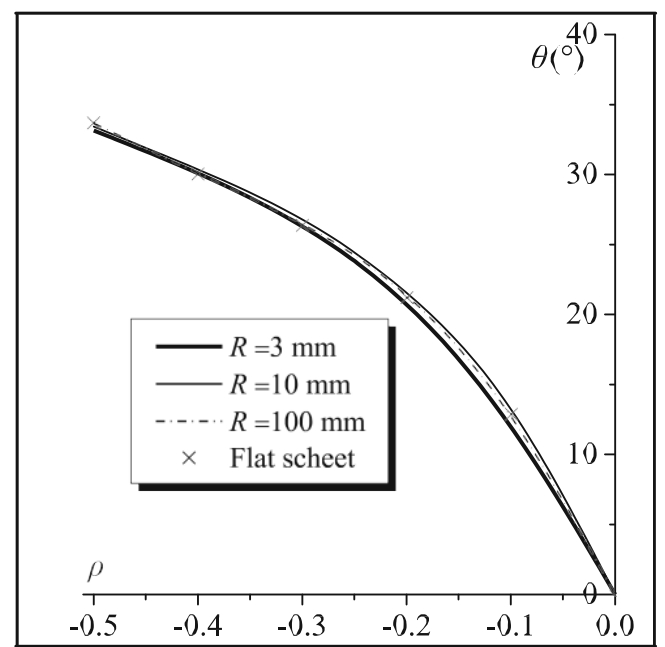

Fig. 6 Effect of the bending radius on the necking band orientation $\theta$ for negative values of the strain-path ratio $\rho$ plotted in terms of the stretching strain components only, and the one represented in terms of the total strain components) coincide exactly when the curvature radius is taken very large (e.g., $\mathrm{R}=100 \mathrm{~mm}$, or a flat sheet).

\section{Effect of normal stress}

The effect of normal stress on the formability of a flat metal sheet is investigated in Fig. 8. The curvature effect is not considered in the results of Fig. 8, since the sheet is assumed to be flat. In this figure, two different values of maximal normal pressure $\alpha$ are used: $\alpha=0 \mathrm{MPa}$ (which correspond to the traditional case of plane-stress state) and $\alpha=200 \mathrm{MPa}$. When $\alpha$ is set to $0 \mathrm{MPa}$, the results displayed in Fig. 7 are naturally recovered. The results of Fig. 8 demonstrate that the application of compressive normal stresses on a sheet allows its formability to be enhanced, especially in the range of positive strain paths. These results are consistent with several earlier investigations (see, e.g., $[10,13,14]$ ).

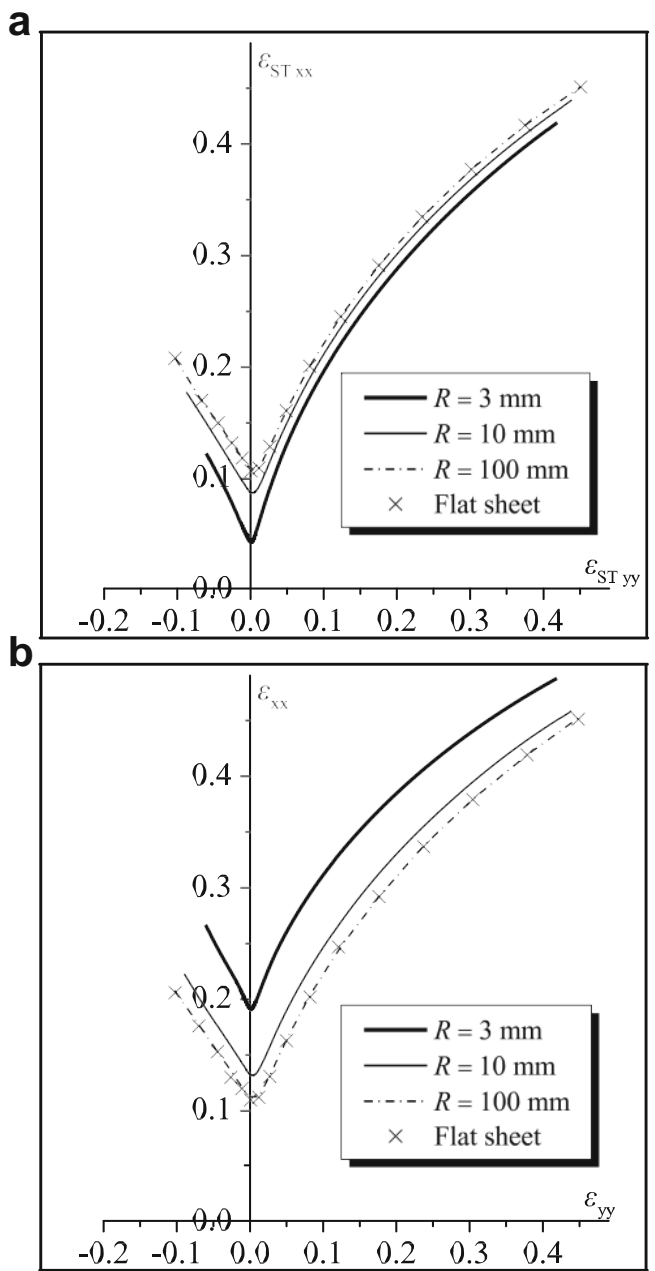

Fig. 7 Effect of the bending radius on the location and the shape of FLDs: (a) FLDs only in terms of stretching strain components; (b) FLDs in terms of total strain components 


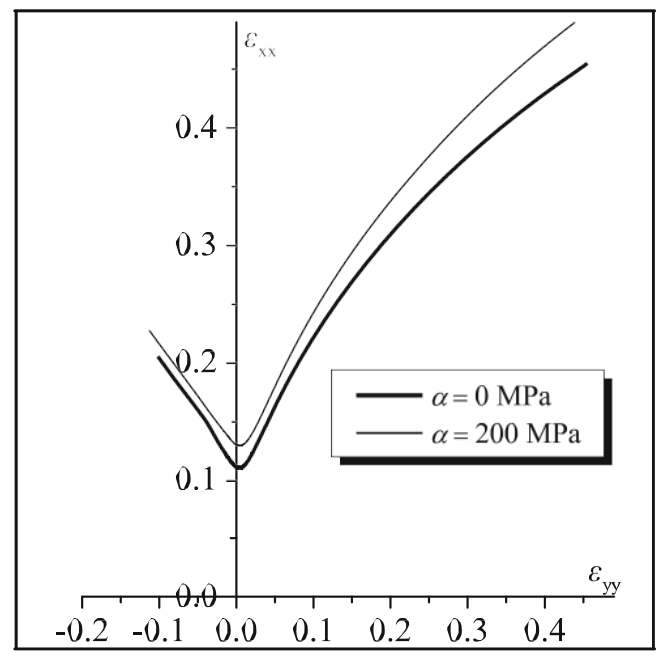

Fig. 8 Effect of normal stress on the location and the shape of FLDs

\section{Combined effects of curvature and normal stress}

The combined effects of bending and normal stress on the enhancement of formability are highlighted in Fig. 9. For instance, the limit strain for a plane strain tensile state is approximately equal to 0.2 when the curvature $\mathrm{R}$ and the maximal pressure $\alpha$ are set to $3 \mathrm{~mm}$ and $200 \mathrm{MPa}$, respectively. However, the limit strain for the same strain path is equal to only 0.1 when the sheet is taken to be flat and under planestress condition (i.e., the classical assumptions for the determination of traditional FLDs). The results of this figure highlight the importance of considering the effects of both curvature and normal stress in the modeling and the prediction of the onset of localized necking. Indeed, the use of classical tools, which are developed under the traditional assumptions (i.e., homogeneity of the deformation through the thickness

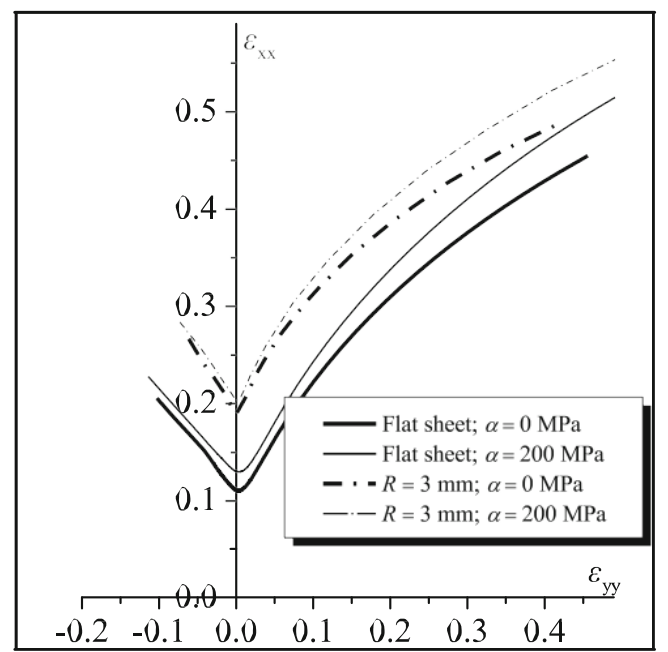

Fig. 9 Combined effects of curvature and normal stress on the location and the shape of FLDs and plane-stress state), for the prediction of formability of some parts and components involved in various industries may lead to inaccurate and inconsistent conclusions.

\section{Concluding remarks}

A numerical tool has been developed in this paper for the prediction of localized necking in sheet metals undergoing combined bending-stretching loadings and submitted to outof-plane compressive stresses. This model may be useful to help understand how the respective and combined effects of bending and normal stresses, which are commonly involved in forming processes, would affect sheet metal formability. Contrary to some existing models, where the effects of curvature and normal stress on formability are investigated separately, the current work allows the two effects to be taken into consideration within the same model. Furthermore, the present model is capable of considering a heterogeneous distribution of normal stresses through the thickness of the sheet. In the current contribution, the mechanical behavior of the sheet metal has been described by the rigid-plastic flow theory, while localized necking prediction is based on the imperfection approach. From the numerical predictions obtained by applying this tool, it is concluded that the addition of bending loading to traditional stretching and/or the consideration of out-of-plane compressive stresses allows significantly delaying the occurrence of localized necking and, thus, enhancing the formability of the studied sheet. The use of the proposed tool, instead of traditional available tools based on the classical assumptions, is believed to provide more accurate predictions for the formability of a number of industrial parts and components.

It is also worth noting that the current work is a first step in a wider project, which aims to investigate the effects of bending and normal stress on the formability of thin metal sheets. In future work, the developed numerical tool will be extended in order to take into account more advanced constitutive models, which would allow a better description of the mechanical behavior of the studied materials. Such elaborate models will include the effects of elasticity, strain-rate sensitivity, plastic anisotropy and kinematic hardening. Besides, the compatibility condition given by Eq. (9) will be written in a local form, accounting for the heterogeneity of the jump vector through the thickness of the sheet. Indeed, in the current version of the model, the jump vector is assumed to be homogeneous through the thickness and independent of the position of the integration points relative to the neutral axis of the sheet. The consideration of the heterogeneity of the jump vector would allow a more precise description of the bendingstretching process and thus a more accurate prediction of the forming limit diagrams. 
Compliance with ethical standards

Conflict of interest The authors declare that they have no conflict of interest.

\section{References}

1. Keeler SP, Backofen WA (1963) Trans ASM 56:25

2. Goodwin GM (1968) Metallurgia Italiana 60:767

3. Marciniak Z, Kuczynski K (1967) Int J Mech Sci 9:609
4. Hutchinson JW, Neale KW, Koistinen DP, Wang NM (Eds.) (1978) Mechanics of Sheet Metal Forming. Plenum 127

5. Shi M, Gerdeen J (1991) J Mater Shap Technol 9:253

6. Sriram S, Yao H, Ramisetti N (2012) J Manuf Sci Eng 134:031003

7. He J, Xia ZC, Li S, Zeng D (2013) J Manuf Sci Eng 135:227

8. He J, Xia ZC, Zeng D, Li S (2013) J Eng Mat Tech 135:031009

9. Safdarian R (2015) Mech Res Commun 67:47

10. Gotoh M, Chung T, Iwata N (1995) JSME Int J Ser A 38:123

11. Swift H (1952) J Mech Phys Solids 1:1

12. Hill R (1952) J Mech Phys Solids 1:19

13. Allwood JM, Shouler DR (2009) Int J Plasticity 25:1207

14. Fatemi A, Dariani BM (2015) Int J Adv Manuf Tech 80:1497 